\title{
A familial polymorphic variant of chromosome $5^{*}$
}

\author{
M SEABRIGHT, N M GREGSON, AND M JOHNSON \\ From the Wessex Regional Cytogenetics Unit, General Hospital, Salisbury, Wiltshire, England
}

SUMMARY Two male fetuses and their mothers, referred for amniocentesis because of high levels of AFP in their blood, were found to carry an anomalous chromosome 5 with a large heterochromatic segment in the long arm. One of the fetuses had an additional ring chromosome.

The presence of heterochromatic segments in autosomes other than 1,9 , and 16 is uncommon. Meyer et $a l^{1}$ described an enlarged $\mathrm{C}$ band extending into the short arm of chromosome 12 in a patient with severe mental retardation. The heteromorphic chromosome was inherited from the father and was found in other relatives. They also reported a case of $17 \mathrm{ph}+$ in a mother of a Down's syndrome child. Three further cases of $12 \mathrm{ph}+$ are reported by Sofuni et al. ${ }^{2}$

We describe here a new heterochromatic variant of chromosome $5 \mathrm{q}$ which was observed in two apparently unrelated families.

* This work was supported by a research grant from the Wessex Regional Health Authority.

Received for publication 21 January 1980

\section{Case reports}

FAMILY A

The index case was a prenatally screened male fetus. The referral for prenatal diagnosis was made because the mother, a 23-year-old primigravida, was found to have a high level of AFP in her serum. The concentration of AFP in the liquor at 19 weeks of gestation was normal.

It is our practice to test for AFP level and examine the fetal chromosomes in all samples of amniotic fluid, irrespective of the indication for the amnios centesis.

The chromosomes were, therefore, analysed and found to be abnormal, with a complement 47,XY,5qh +, +ring(?) (fig 1). The maternal karyo-

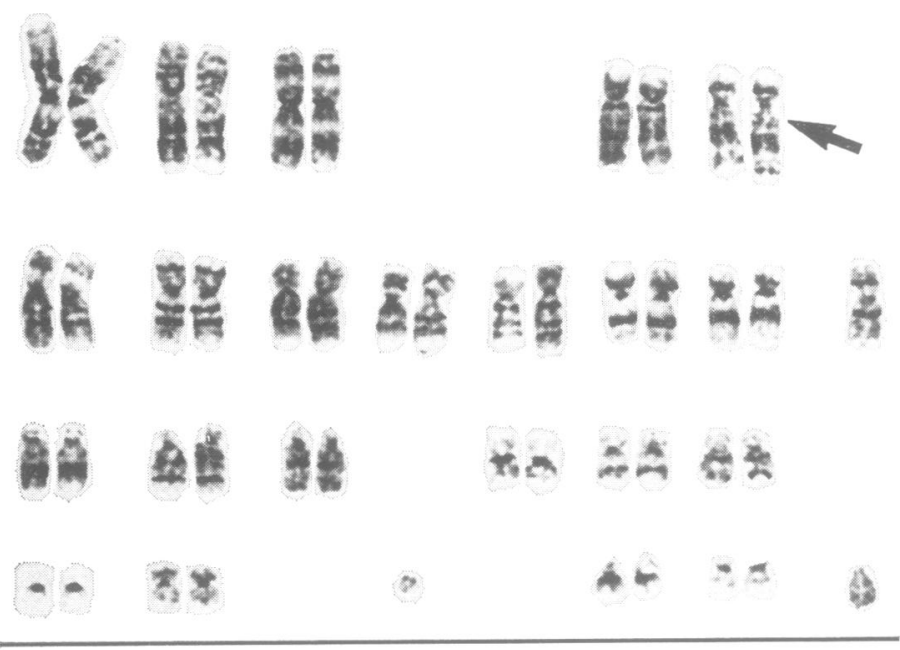

FIG $1 G$ banded karyotype showing the anomalous chromosome 5 and rings from eight metaphases (index case, family $A$ ).

Rings from 8 metaphases 


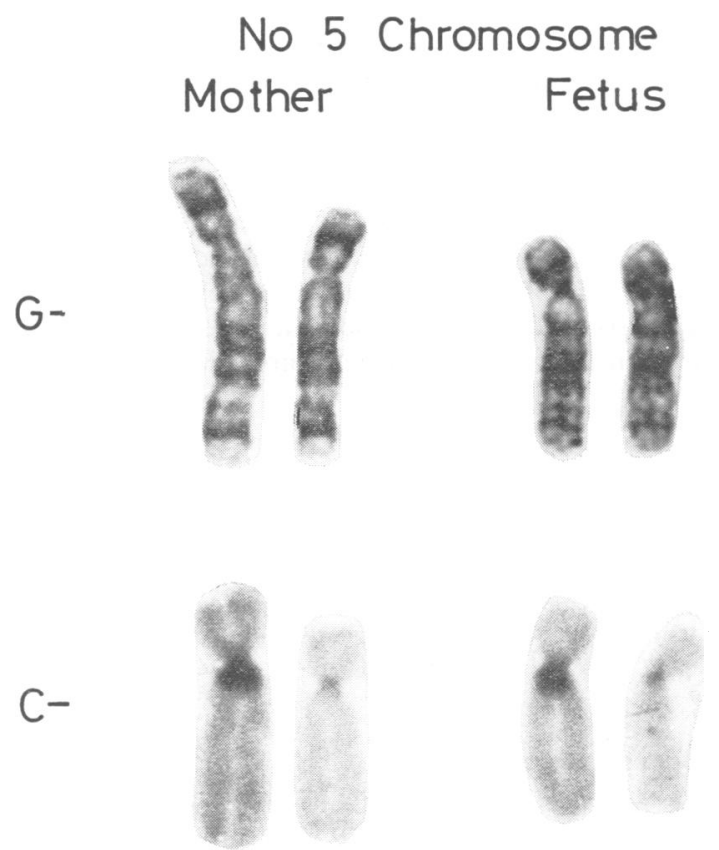

FIG $2 G$ and $C$ banded partial karyotype of mother and fetus (family $A$ ).

type showed the presence of $5 \mathrm{qh}+$ (fig 2). Family studies showed that the mother's sister and their father also carried the variant 5 (fig 3). The presence of the extra ring chromosome was considered to be a high risk to the fetus and this pregnancy was terminated. The fetus had a large mouth and micrognathia with no other apparent abnormalities.

FAMILY B

The index case was also a prenatally screened male fetus. The mother was a 24-year-old primigravida.
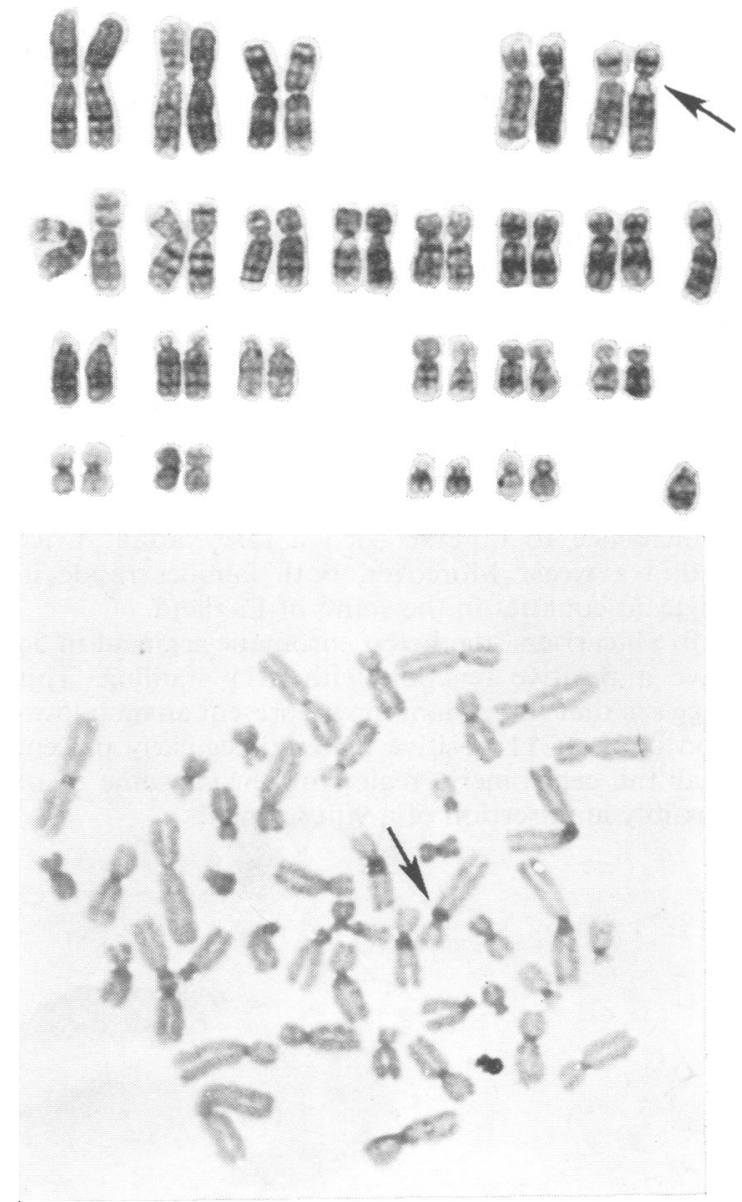

FIG $4 \quad G$ banded karyotype (top) and $C$ banded metaphase (bottom) (index case, family B), $5 q h+$ at arrow.

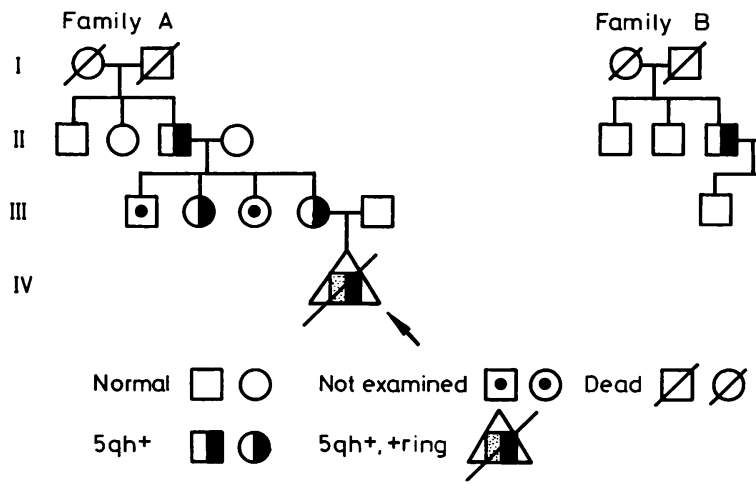

FIG 3 Pedigrees of family $A$ and $B$. 
The indication for amniocentesis was an abnormally high level of AFP in her serum, but this was found to be within normal limits in the amniotic fluid at the 18th week of gestation. The karyotype of the fetus was $46, \mathrm{XY}, 5 \mathrm{qh}+$ (fig 4). The variant chromosome 5 was of maternal origin and also present in her father (fig 3).

This pregnancy continued, resulting in the birth of a phenotypically normal infant.

\section{Discussion}

Extensive enquiries have so far failed to establish a relationship between these two families, but it is highly probable that they may share a common ancestor. Otherwise, it would be an extraordinary coincidence to observe such a rare variant twice within a week. Moreover, both families reside in adjacent counties in the south of England.

In all carriers, the heterochromatic segment in $5 q$ gave a positive reaction with G11 staining. This suggests that the variant may represent an amplification of the G11 positive material regularly present near the centromeric region of chromosome 5 , or possibly an insertion of a $9 \mathrm{qh}$ segment.
The ring chromosome could not be identified using G, C, Q, G11, and NOR banding. It varied in shape and size in different cells. There is insufficient evidence to show whether or not the presence of polymorphic variants predispose their carrier to produce abnormal gametes. The presence of the extra ring in our case may have been fortuitous. There is no known history of reproductive problems in either family. Nevertheless, we feel that prenatal diagnosis is advisable whenever a patient is known to carry an unusual type of heteromorphic variant.

\section{References}

1 Meyer J, Matsuura P, Jacobs P. Inversions and other unusual heteromorphism detected by C-banding. Hum Genet $1978 ; 45: 43-50$.

2 Sofuni T, Tanabe K, Ohtaki K, Shimba H, Awa AA Two new types of C-band variants in human chromosomes Jpn J Hum Genet 1974;19:251-6.

Requests for reprints to Dr Marina Seabright, Wessex Regional Cytogenetics Unit, The General Hospital, Salisbury, Wilts SP2 7SX. 